\title{
Transitioning children with urological issues into adulthood
}

\author{
Peter A.M. Anderson, MD; Ashley Cox, MD
}

Department of Urology, Dalhousie University, Halifax, NS, Canada

Cite as: Can Urol Assoc J 2018;12(4Suppl1):S1. http://dx.doi.org/10.5489/cuaj.5312

t could be said that pediatric urologists (and their patients) have fallen victim to their own success. With advances

in surgical technique and medicine in general, the child with a urological issue should expect to live a long and fruitful life, hopefully cured of the original issue. However, as pointed out in the hypospadias, exstrophy and urinary diversion articles, the risk of long-term complications is never completely eliminated. Therefore, even after the pediatric urologist has discharged the patient from care and he has entered into the adult domain, issues may re-emerge or develop de novo. The cancer survivor is an often overlooked example of this phenomenon. We also have the scenario where the condition never resolves, but rather evolves as the patient ages, as discussed in the articles on varicocele, posterior urethral valves, and dysfunctional voiding.

This collection of articles on various topics pertinent to the transition of children with urological issues to adulthood fills a gap that has historically existed. The target audience is the general urologist, although we hope that pediatric and adult subspecialists will find some useful perspectives as well. Each article has a pediatric and an adult urologist author who is an expert on the topic, with deliberate intertwining of their respective views. We have used clinical vignettes in an attempt to make each topic as relevant as possible to the practicing urologist, keeping in mind that not every point can be drawn directly from such cases. We have avoided discussion of esoteric points relevant only to subspecialists in a specific field.

Throughout the process of creating this series, we have challenged ourselves to think about not only what can be done, but what should be done for a particular patient. Some of the conditions discussed are relatively common, while others are likely to be encountered rarely in a general urology practice, but in all cases we must strive to serve our patients to the best of our ability. We hope that the reader finds a few kernels of wisdom to help in the management of these challenging scenarios; we certainly did.

Correspondence: Dr. Peter A.M. Anderson, Department of Urology, Dalhousie University, Halifax, NS, Canada; Peter.Anderson@iwk.nshealth.ca 